\title{
Modelling lateral width for merging of U-turning vehicles in divided urban roads
}

\author{
Tathagatha Khan and Smruti Sourava Mohapatra* \\ Department of Civil Engineering, Indian Institute of Technology (Indian School of Mines), Dhanbad 826 004 , India
}

\begin{abstract}
The present study was carried out to understand the lateral width for merging (LWM) characteristics and for developing LWM prediction models for U-turning vehicles. Data were collected from 14 test sections using videography. The collected data were analysed and it was observed that various operational characteristics, namely service delay, occupancy time and approaching through traffic volume influence LWM of U-turning vehicles. Subsequently, utilizing the stepwise multilinear regression method, LWM prediction models were developed in six-lane and four-lane divided urban roads. Finally, using the proposed $\mathrm{LWM}_{85}$ prediction model, a geometrical augmentation scheme has been suggested. The present study holds significance to enhance the level of service and safety at uncontrolled mid-block median openings.
\end{abstract}

Keywords: Geometrical augmentation, heterogeneous traffic, lateral width for merging, mid-block median opening, U-turning vehicles.

INDIA has experienced a swift expansion of economic activities, which has resulted in an enormous surge in vehicular traffic. According to recent reports, the country has 210 million registered motor vehicles ${ }^{1}$ and a road network of around 5.5 million kilometres ${ }^{2}$. Therefore, construction of multi-lane roads in urban areas is indispensable to cater to the needs of escalating vehicular traffic. Multi-lane roads are commonly constructed with a raised median to separate the opposing traffic movements $^{3}$. The primary function of the raised median is to evade head-on collision among vehicles moving in the opposite direction, and to channelize traffic into streams at intersections which are considered a vital part of any road network ${ }^{4}$. Openings are provided in these raised medians for provision of taking U-turns; these are called mid-block median openings (MBMOs). At these MBMOs, only two types of movement are possible, namely through movement and U-turn movement. The former has the priority and is known as major priority movement, while the latter is considered a minor priority movement. These MBMOs in a developed country are generally provided with stop and yield signs, and operate as controlled intersections. On the contrary, in India, these signs are not

*For correspondence. (e-mail: smruti@iitism.ac.in) followed by U-turning drivers. The drivers habitually disobey the rule of priority and frequently reverse the priority of movement in mixed traffic condition ${ }^{5,6}$.

Furthermore, in a heterogeneous traffic system, the vehicles operate differently compared to the homogeneous traffic condition due to disparity in the operating and performance characteristics of the various vehicle categories. Traffic in the road network is comprised of fast- and slow-moving vehicles, and the static and dynamic characteristics of vehicles possess wide disparity. Moreover, the same right of way is shared by all categories of vehicles because traffic streams are not segregated on the basis of vehicle type. In the prevailing situation, smaller-sized vehicles often squeeze through any available gap between large-sized vehicles and move in a disorganized manner ${ }^{7}$. In mixed traffic conditions, vehicles with varying static dimensions are found to use the same space of carriageway width. In these peculiar conditions as prevailing in India, traffic operation at these MBMOs often becomes chaotic. The compromised traffic operation at these locations results in problems such as reduction in capacity and deterioration in traffic operating conditions ${ }^{8}$. Khan and Mohapatra ${ }^{9}$ studied the effect of various characteristics, namely gender and vehicle characteristics, on lateral merging position of U-turning vehicles and observed that male drivers are more aggressive and require less carriageway width compared to female drivers. Moreover, personal and loaded vehicles required more carriageway width and also took turns cautiously compared to commercial and empty vehicles.

Capacity estimation is the first and important exercise in the planning of any traffic facility ${ }^{10}$. Many researchers have contributed significantly to the evaluation of capacity of different traffic facilities such as uncontrolled intersections and minor street stop-controlled intersections ${ }^{11-13}$. Estimation of capacity at MBMOs is also essential. One of the earliest studies on U-turn capacity was carried out by Al-Masaeid ${ }^{14}$, who estimated the same by gap acceptance and empirical methods. Traffic performance and U-turn capacity have been studied by various researchers ${ }^{15-}$ ${ }^{17}$. Jenjiwattanakul and Sano ${ }^{15}$ studied the effect of waiting time on U-turning vehicles at MBMOs. They reported that U-turning vehicles get frustrated and tend to accept smaller gaps when they have to wait for more than $30 \mathrm{sec}$ at MBMOs. Additionally, they suggested traffic control and management at MBMOs, considering safety as a 
major concern ${ }^{15}$. Liu et al. ${ }^{16}$ developed a procedure for capacity estimation of U-turning vehicles at MBMOs. Maximum likelihood and linear regression models were used to estimate the critical gap and follow-up time for U-turning vehicles respectively. The authors used Harder's model to estimate U-turn capacity, which requires three parameters, namely critical gap, follow-up time and conflicting traffic volume (CTV) ${ }^{16}$. Harder's model result was tested in the field using Kyte's method and observed to provide a satisfying outcome. Furthermore, the simulation program VISSIM has been employed in some studies for the evaluation of capacity ${ }^{18,19}$. Liu et al. ${ }^{18}$ proposed a procedure for modelling U-turn movement at MBMOs using VISSIM. Behavioural features of U-turning drivers were determined by field data, which were collected from 13 locations. The authors calibrated and validated the VISSIM simulation model using a genetic algorithm procedure. The results were compared with field capacity and observed to yield a MAPE value of $17.6 \%$ for four-lane and $20.7 \%$ for six-lane roads ${ }^{18}$. A critical review of the literature shows that capacity estimation of uncontrolled MBMOs requires three factors, viz. follow-up time, critical gap and CTV ${ }^{10}$.

The estimation of CTV in heterogeneous traffic conditions is difficult due to the uniqueness of mixed traffic governing in developing countries in general and India in particular. In a study carried out at Florida, USA, Liu et $a .^{20}$ estimated that CTV for U-turns on a six-lane road is 2.2 times the average ATTV in each lane. However, these findings do not hold significance under Indian conditions as the traffic is heterogeneous in nature, and the rule of priority is often violated ${ }^{5}$. In heterogeneous traffic conditions, different types of vehicles varying in static and dynamic characteristics use the same carriageway width, whereas in homogeneous traffic conditions separate lanes are provided for different types of vehicles. The problems of heterogeneity in traffic justify the need for studies on lateral width for merging (LWM) for better estimation of CTV for U-turning traffic.

\section{LWM of vehicles taking U-turns}

The U-turning manoeuvre is a risky process as there is a chance of conflict with the approaching through traffic (ATT) at MBMOs. The amount of conflict depends upon the area encroached by a U-turning vehicle. Hence we have proposed the LWM parameter in the present study. This is defined as the lateral distance required by the U-turning traffic to complete its merging process at the MBMO area. LWM has been measured in the field by collecting placement data of the most critical wheel while traversing at the MBMO. Hence, the present study was undertaken with the following objectives.

- To understand the LWM characteristics of different categories of U-turning vehicles at MBMOs.
- To assess the effect of service delay (SD), occupancy time (OT), and ATTV on LWM of U-turning vehicles.

- To develop LWM prediction models using operational characteristics data for different categories of vehicles in four-lane and six-lane divided roads.

- To recommend an geometrical augmentation scheme for enhancing traffic operation at MBMO.

The full outline of the present study is given in Supplementary Figure 1.

\section{Study area}

In the present study we have evaluated study LWM of U-turning vehicles and the effects of various traffic operational characteristics on it at MBMOs. Traffic data were collected from 14 MBMOs in multi-lane urban roads from different cities of India. All the data-collection sites were completely uncontrolled without any sign, signal or deployment of police personnel. The data collection sites were finalized in such a way that the influence of onsite parking, pedestrian movement, presence of adjacent intersection and friction was nominal. The MBMOs were located in four states, viz. Assam, Jharkhand, Karnataka and Odisha. The geographic locations of the datacollection sites are presented in Supplementary Figure 2. Among the 14 MBMOs, 7 were in the six-lane divided roads and the remaining 7 sites in four-lane divided roads. All the six-lane divided test sections had a raised kerb with a carriageway width of around $9.5 \mathrm{~m}$, whereas the four-lane test sections had a wide shoulder with a carriageway width of around $7.5 \mathrm{~m}$ in each direction.

Road geometry details, along with the data collection date and duration of all 14 test sections are given in Supplementary Table 1. The observed traffic count and composition of U-turning and ATT are also given in the Supplementary Tables 2 and 3 respectively. In this study, all the U-turning vehicles have been separated into six categories, viz. motorized two-wheelers (2Ws), motorized three-wheelers (3Ws), small cars (SCs), big cars (BCs), light commercial vehicles (LCVs) and heavy vehicles (HVs). SCs refer to cars having a length of less than $4 \mathrm{~m}$, whereas BCs refer to cars having a length of more than $4 \mathrm{~m}$ (refs 21 and 22).

In this study, LWM of the front left wheel was considered as critical as it traverses the outer path ${ }^{5}$, whereas for $3 \mathrm{Ws}$, the rear left wheel was measured since the configuration is peculiar with a front central wheel. The entire method to collect LWM data of vehicles taking U-turns is presented in detail elsewhere ${ }^{22}$. Simultaneously, traffic operational data were collected using video recording at MBMOs. The traffic data were collected by setting up a high-definition video recorder at a vantage point using a tripod to have an unobstructed sight of the 

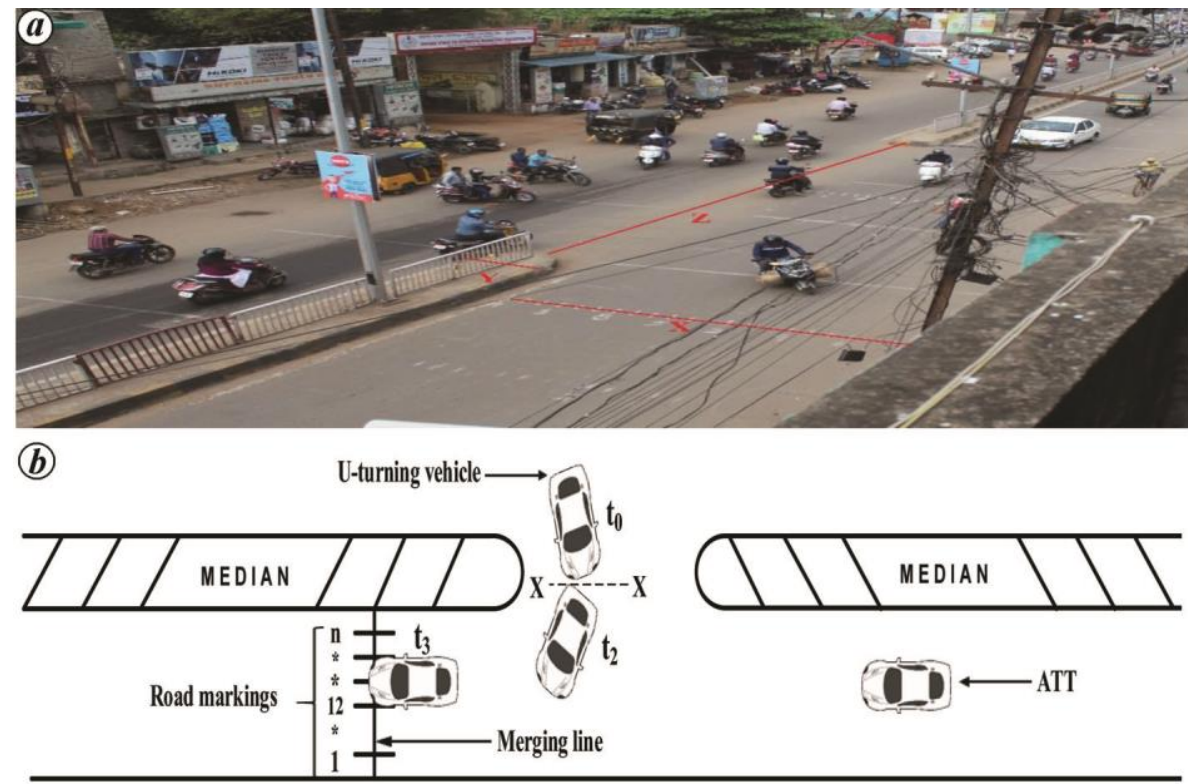

Figure 1. Layout of a typical mid-block median openings (MBMO). $\boldsymbol{a}$, Schematic diagram of MBMO showing road markings. $\boldsymbol{b}$, Snapshot of test section showing road markings. $x$, Rod width; $y$, Median width; $z$, Width of median opening.
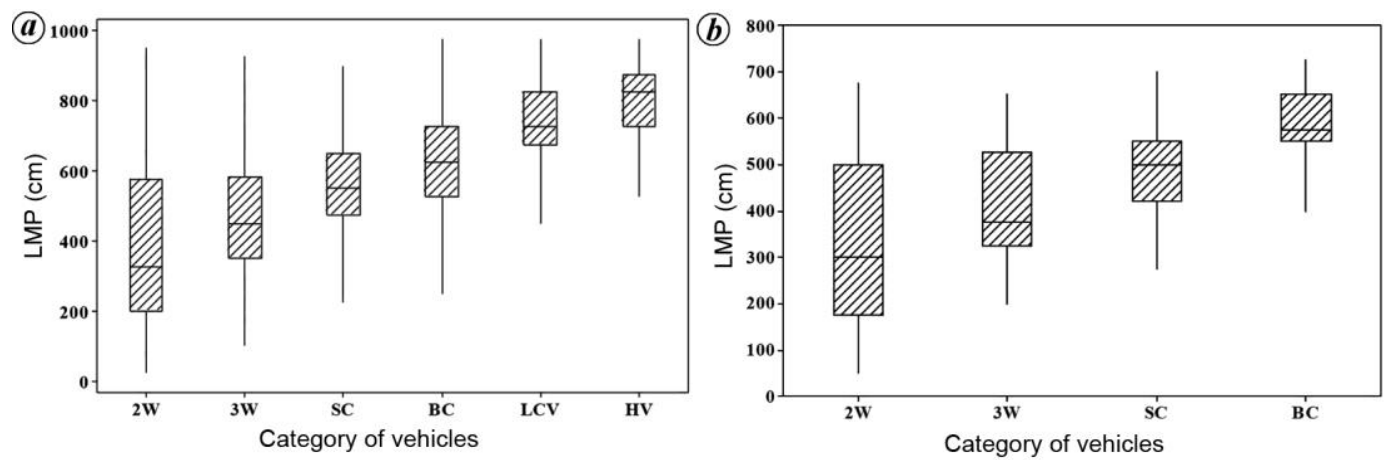

Figure 2. Box plots of lateral width for merging (LWM) data; (a) Six-lane road and (b) four-lane road.

MBMO area. Figure $1 a$ presents a view of the data collection site. Traffic data were collected using a video camera with 25 frames per second (fps). The data were collected only on weekdays from 7 a.m. to 5 p.m. and during pleasant weather, without any traffic jam.

\section{Data extraction}

The recorded videos were played several times in the laboratory on a large display monitor for extracting the required traffic data using video image-processing software. Various parameters pertaining to the traffic operational characteristics at the MBMOs, namely LWM of U-turning vehicles, their composition and volume, and ATT, SD and OT were extracted systemically.

Figure $1 b$ explains the data extraction process. From the figure, it is evident that the wheel touches the line marked as 12. Hence for that particular vehicle, LWM is $300(12 * 25)$ from the kerb. Whenever a vehicle needs to take a U-turn, first, it searches for a suitable gap in the ATT stream. If the gap is sufficient to take a U-turn, then the vehicle proceeds into the MBMO area and completes the manoeuvre. If not, the vehicle stops at the MBMO area and waits till a suitable gap is available. The time spent by the vehicle waiting for the gap is considered as $\mathrm{SD}^{23}$. In this study, a reference line has been used for the estimation of SD. This line is the stop line where Uturning vehicles stop before encroaching into the MBMO area (Figure $1 b)$. SD is measured from the time $\left(t_{0}\right)$ of arrival of the front bumper of a U-turning vehicle at the reference line $(X-X)$ to the time $\left(t_{2}\right)$ of departure of the rear bumper of the vehicle off the reference line $(X-X)$ (Figure $1 b$ ). SD is calculated as follows

$$
\mathrm{SD}=t_{2}-t_{0}
$$




\section{RESEARCH ARTICLES}
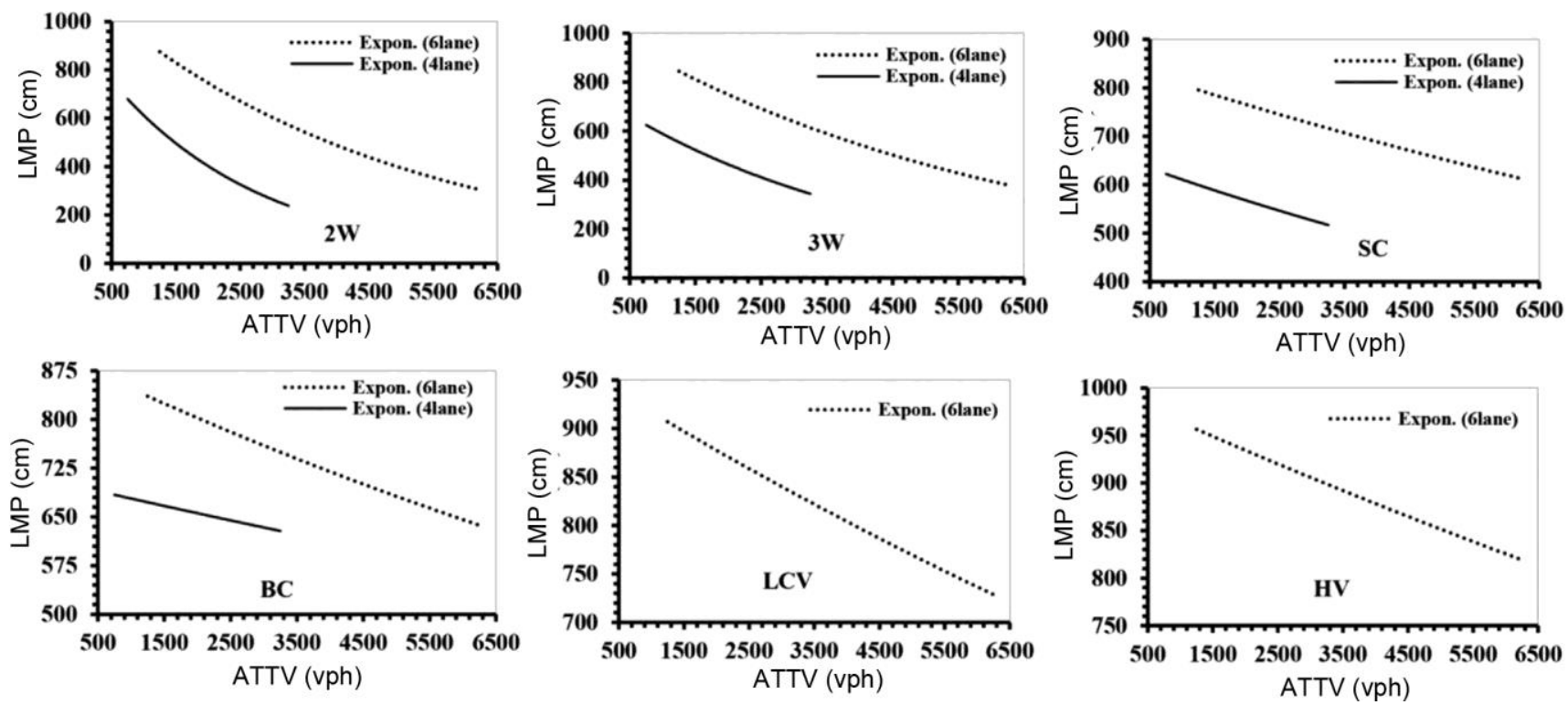

Figure 3. Disparity of $\mathrm{LWM}_{85}$ at varying ATTV (approaching through traffic volume) in six-lane and four-lane roads.

Likewise, the occupancy time (OT) of a U-turning vehicle starts when the front bumper departs from the reference line and ends when the back bumper departs the merging line. From field studies, it was found that the position of the merging line varies for different categories of U-turning vehicles. OT was measured from the time departure of $\left(t_{1}\right)$ of the front bumper of the U-turning vehicle from the reference line $(X-X)$ to the time of departure $\left(t_{3}\right)$ of the back bumper of the vehicle from the merging line.

$$
\mathrm{OT}=t_{3}-t_{1}
$$

\section{Analysis of data}

The collected field data of 14 test sections were analysed to get an insight into the characteristics of LWM of each vehicle category making a U-turn. LWM of all the vehicle categories considered here was studied independently. As mentioned previously, LWM of vehicles was recorded from the kerbside for ease of data collection, but for analysis, the median was considered as the reference point and LWM of an individual vehicle was reported. The recorded LWM of the individual vehicles was subtracted from the carriageway width and reported from the median side. Subsequently, various statistical parameters pertaining to LWM were computed and compiled in the form of a box plot (Figure 2). The perpendicular axis displays the range of LWM data ${ }^{24}$. The bottom box represents the 25th percentile of LWM data and the top box represents the 75 th percentile of $\mathrm{LWM}$ data ${ }^{25}$. Moreover, the dividing line between the two boxes represents the 50th percentile of LWM data. Wide variation in LWM at six-lane and four-lane roads among all the vehicles considered in this study can be observed in Figure $2 a$ and $b$ respectively. The 50th percentile of LWM was minimum for $2 \mathrm{Ws}$, followed by $3 \mathrm{Ws}$, SCs, BCs, LCVs and HVs in the six-lane and four-lane roads.

Figure 2 indicates that the mean LWM of $2 \mathrm{Ws}$ is commonly found to be located on the lane beside the median. This is due to the small turning radius and dimension of $2 \mathrm{Ws}$ compared to other vehicles. Furthermore, the peculiar driving behaviour of $2 \mathrm{~W}$-riders allows them to complete a turning manoeuvre using the lane beside the median. Likewise, $3 \mathrm{~W}$-drivers are considered to have an aggressive driving nature ${ }^{26}$. Due to this $3 \mathrm{~W}$, ATT drivers are forced to reduce their speed to allow $3 \mathrm{Ws}$ to complete the U-turn manoeuvre. The frontal conical shape and small turning radius in comparison to the cars allow $3 \mathrm{Ws}$ to complete their merging using lesser width of the carriageway. The average LWM of SCs and BCs was found to be higher than that of $3 \mathrm{Ws}$, because SCs and BCs are wider than $3 \mathrm{Ws}$. From the above-mentioned characteristics of SCs and BCs, their average LWM values were observed to be comparatively higher than $3 \mathrm{Ws}$. Likewise, HVs have a high turning radius due to their long wheelbase. Therefore, the observed LWM is mostly towards the kerbside. From Figure 2, it is also evident that LWM of $2 \mathrm{Ws}$ varies widely compared to all other vehicles considered in the study. This is attributed to the fact that LWM is not channelized because of the small size of $2 \mathrm{Ws}$ and inconsistent behaviour of the drivers. LWM of HVs is commonly found to be more concentrated towards the lane beside the kerbside due to higher turning radius and large physical dimensions. Hence it can be inferred that small-sized vehicles have the flexibility to explore the entire carriageway width in the opposite direction, whereas larger vehicles are not able to use the lane near the median side. 


\section{5th percentile lateral width for merging}

As discussed in the previous section, wide variation in LWM was observed for all the six categories of vehicles considered in the present study. LWM varies not only with vehicle category, but also with carriageway width. Furthermore, within the same category of vehicles also wide variation has been observed. Therefore, a statistical parameter known as 85th percentile lateral width for merging $\left(\mathrm{LWM}_{85}\right)$ has been proposed in this study to model the LWM characteristics with varying traffic operational attributes. There are two reasons for choosing $\mathrm{LWM}_{85}$ for describing the LWM characteristics of Uturning vehicles. The first is that the 85 th percentile is a widely accepted statistical parameter to describe a traffic phenomenon $^{27}$. Moreover, traffic engineers have used 85th percentile speed for setting up the speed limit for traffic in the roadways ${ }^{27}$. The second reason for choosing $\mathrm{LWM}_{85}$ is that the modelling of LWM characteristics has been carried out to develop placement prediction models. These prediction models for all the six vehicle categories considered in the present study have been utilized to suggest a geometrical augmentation scheme in the MBMO area. For this, we need to overestimate the width of the conflict area at the median opening.

\section{Modelling $L W M_{85}$ with operational characteristics}

In this study, we have modelled the relationship of $\mathrm{LWM}_{85}$ with different operational characteristics, namely SD, OT and ATTV. In order to obtain the hourly ATTV, 5 min volume count of the recorded video was carried out $^{28}$. For modelling, the extracted data were combined on the basis of similar hourly ATTV. After combining the data with similar ATTV, the traffic operational parameters were estimated. From the aggregated data, $\mathrm{LWM}_{85}$, ATTV and average SD as well as OT were calculated for all categories of vehicles. In the following sections, the effect of operational characteristics of different vehicle categories on $\mathrm{LWM}_{85}$ for six-lane and four-lane divided urban roads are discussed.

Influence of ATTV on $L W M_{85}$ : Figure 3 presents the variation of $\mathrm{LWM}_{85}$ with ATTV in six-lane and four-lane roads. Table 1 shows the $\mathrm{LWM}_{85}$ values for different types of U-turning vehicles at varying ATTV. From the figure and table, it can be observed that $\mathrm{LWM}_{85}$ has a tendency to shift towards the lane adjacent to the median with increase in ATTV. This is because, at low ATTV, a U-turning vehicle has the freedom to merge freely using the maximum width of the road. Whereas with an increase in ATTV, the U-turning vehicle cannot merge freely because of ATT. Due to this increased possible conflict, it is obvious that the U-turning vehicle has to wait for a longer period than usual at high ATTV. This makes the driver impatient, and he tries to merge swiftly using less width of the road. This trend has been observed for both six-lane and four-lane roads. In four-lane roads the LWM of $\mathrm{HV}$ is found to be skewed towards the kerbside, and there is not much lateral shift in placement with increase in ATTV. The variation in $\mathrm{LWM}_{85}$ of LCVs and HVs was observed to be 731-885 cm and 833 $950 \mathrm{~cm}$ from the median respectively. SCs and BCs showed variation of around $175 \mathrm{~cm}$ in $\mathrm{LWM}_{85}$ due to variation in ATTV. For four-lane roads, variations in $\mathrm{LWM}_{85}$ for SCs and BCs were less compared to six-lane roads. This is because of the difference in the width of the carriageway. In six-lane roads, SCs and BCs squeeze towards the median with an increase in ATTV, but in four-lane roads they do not have the freedom to squeeze due to restricted carriageway width. A similar observation has also reported in the literature ${ }^{10}$.

Influence of $S D$ on $L W M_{85}$ : Figure 4 presents variations of $\mathrm{LWM}_{85}$ with SD in four-lane and six-lane roads. From the figure, it can be noted that SD of $2 \mathrm{Ws}$ varies from 2.33 to $3.14 \mathrm{sec}$ in a six-lane roads. Likewise, the variation in $\mathrm{SD}$ in four-lane roads is from 2.41 to $4.87 \mathrm{sec}$. Corresponding to $\mathrm{SD}, \mathrm{LWM}_{85}$ was found to vary from 800 to $350 \mathrm{~cm}$ in six-lane road, and from 625 to $245 \mathrm{~cm}$ in four-lane roads. For both six-lane and for four-lane roads, it was observed that $\mathrm{LWM}_{85}$ decreases with increase in SD for 2Ws. Similar trends were observed for all the categories of vehicles. This decreasing trend of $\mathrm{LWM}_{85}$ is because when U-turning drivers experience more delay, they become impatient. This leads to aggressive driving. As a result, the drivers move into the median opening area and try to complete the U-turn utilizing minimal road space. Due to such aggressive driving, the approaching through vehicles many a times reduce their speed or change lanes or both. Such aggressive manoeuvre predominantly occurs in six-lane roads due to the availability of road space. It is a challenge in four-lane roads due to constrained road width.

Influence of OT on $L W M_{85}$ : The effect of OT on $\mathrm{LWM}_{85}$ of U-turning vehicles was evaluated in this study. From Figure 5, it can be observed that OT of $2 \mathrm{Ws}$ varies from 2.74 to $3.70 \mathrm{sec}$ in six-lane roads, and from 2.84 to $4.00 \mathrm{sec}$ in four-lane roads. From the graphical comparison, it can be concluded that the four-lane vehicles take more time to merge than the six-lane vehicles. This is due to the fact that four-lane vehicles merge gradually. During gradual merging, the U-turning vehicles wait at the median opening for sufficient gap, and then start rolling at a very low speed ${ }^{23}$. During this time period, the vehicles accelerate and decelerate several times before merging. The turning speed of four-lane vehicles is also less than that of six-lane vehicles ${ }^{10}$. Hence due to gradual merging and less turning speed OT 
Table 1. Lateral width for merging $\left(\mathrm{LWM}_{85}\right)$ values for U-turning vehicles at varying ATTV

\begin{tabular}{lcllllll}
\hline & \multicolumn{7}{c}{ LWM $_{85}$ of U-turning vehicles } \\
\cline { 3 - 8 } ATTV (vph) & Number of lanes & $2 \mathrm{~W}$ & $3 \mathrm{~W}$ & $\mathrm{SC}$ & $\mathrm{BC}$ & LCV & HV \\
\hline $1000-1500$ & Six-lane & 800 & 775 & 800 & 825 & 885 & 950 \\
$1501-2000$ & & 775 & 766.25 & 775 & 800 & 875 & 950 \\
$2001-2500$ & & 775 & 755 & 775 & 800 & 875 & 950 \\
$2501-3000$ & 725 & 750 & 752.5 & 800 & 875 & 950 \\
$3001-3500$ & 625 & 700 & 725 & 798.75 & 875 & 895 \\
$3501-4000$ & 540 & 675.75 & 700 & 750 & 825 & 850 \\
$4001-4500$ & 380 & 610 & 625 & 650 & 773.35 & 833.75 \\
$4501-5000$ & & 358.75 & 600 & 623.75 & 650 & 752.5 & 836.25 \\
$5001-5500$ & 343.75 & 615 & 633.75 & 650 & 731.25 & 837.5 \\
$5501-6000$ & 350 & 625 & 647.5 & 665 & 750 & 850 \\
$6001-6500$ & & 350 & 643.5 & 650 & 675 & 750 & 850 \\
$500-1000$ & Four-lane & 625 & 600 & 625 & 685 & - & - \\
$1001-1500$ & & 600 & 585 & 616.25 & 675 & - & - \\
$1501-2000$ & & 550 & 575 & 597.5 & 640 & - & - \\
$2001-2500$ & & 300 & 350 & 500 & 580 & - & - \\
$2501-3000$ & & 250 & 375 & 525 & 615 & - & - \\
$3001-3500$ & & 245 & 365 & 525 & 625 & - & - \\
\hline
\end{tabular}
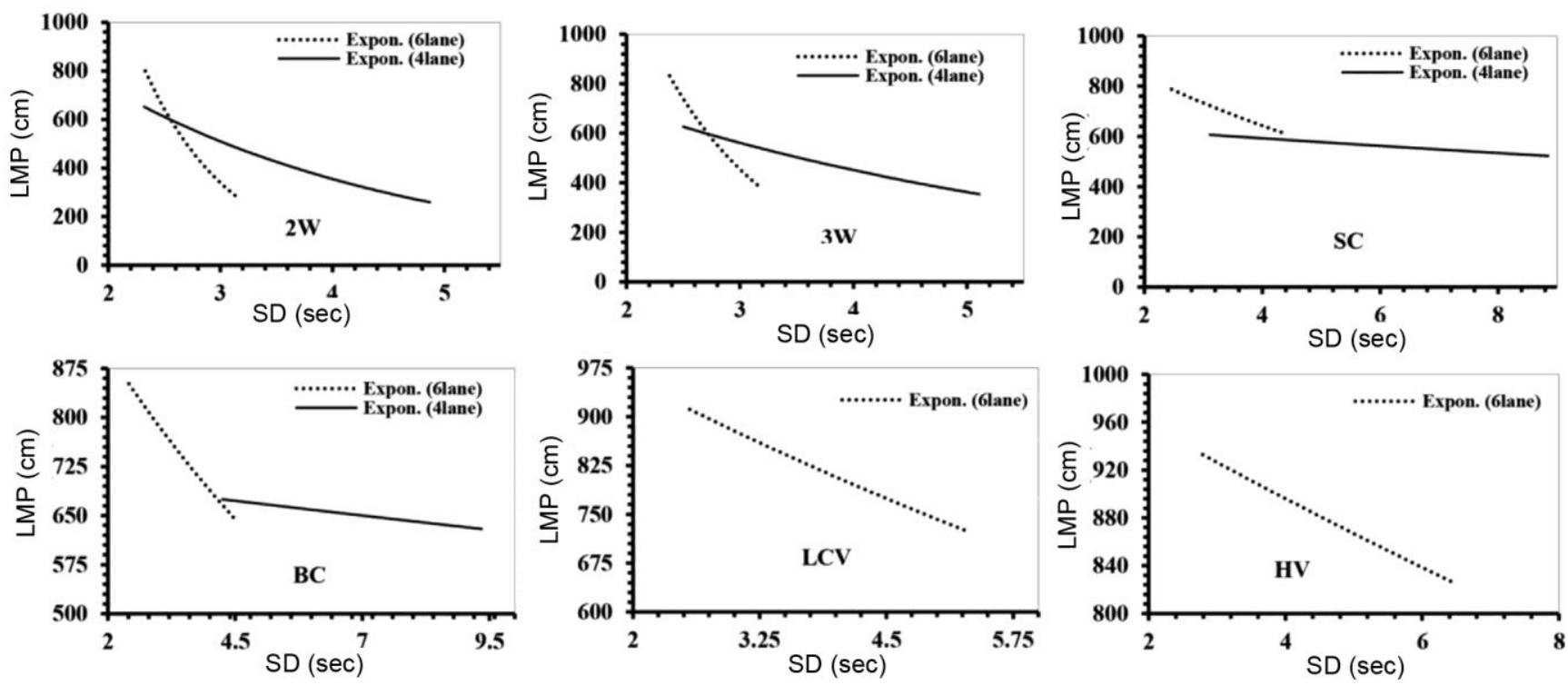

Figure 4. Disparity of $\mathrm{LWM}_{85}$ at varying service delay in six-lane and four-lane roads.

of U-turning vehicles in four-lane roads is more than those in six-lane roads. We studied the variation of LWM $_{85}$ with OT. From Figure 5, it can be observed that $\mathrm{LWM}_{85}$ varies from 350 to $800 \mathrm{~cm}$ in six-lane roads, and from 245 to $625 \mathrm{~cm}$ in four-lane roads. For both six-lane and four-lane roads, it was observed that $\mathrm{LWM}_{85}$ decreases with decrease in OT for 2 Ws (i.e. $\mathrm{LWM}_{85}$ shifts towards the median side with a decrease in OT). A similar trend was observed for all vehicle categories. LWM $_{85}$ shows decreasing trend with increase in ATTV because when the LWM of a U-turning vehicle is nearer to the kerb, the vehicle traverses more distance while taking U-turn. Hence as the U-turning vehicle travels more dis- tance before merging with ATT, it occupies the MBMO area for longer, and therefore OT is observed to be more when $\mathrm{LWM}_{85}$ is more.

\section{$L W M_{85}$ prediction model development}

Modelling of $\mathrm{LWM}_{85}$ has been carried out by considering three independent variables, namely ATTV, SD and OT. A stepwise multiple linear regression using SPSS was performed to develop the proposed $\mathrm{LWM}_{85}$ model for U-turning vehicles at MBMOs. From multicollinearity analysis, no significant correlation was found between 

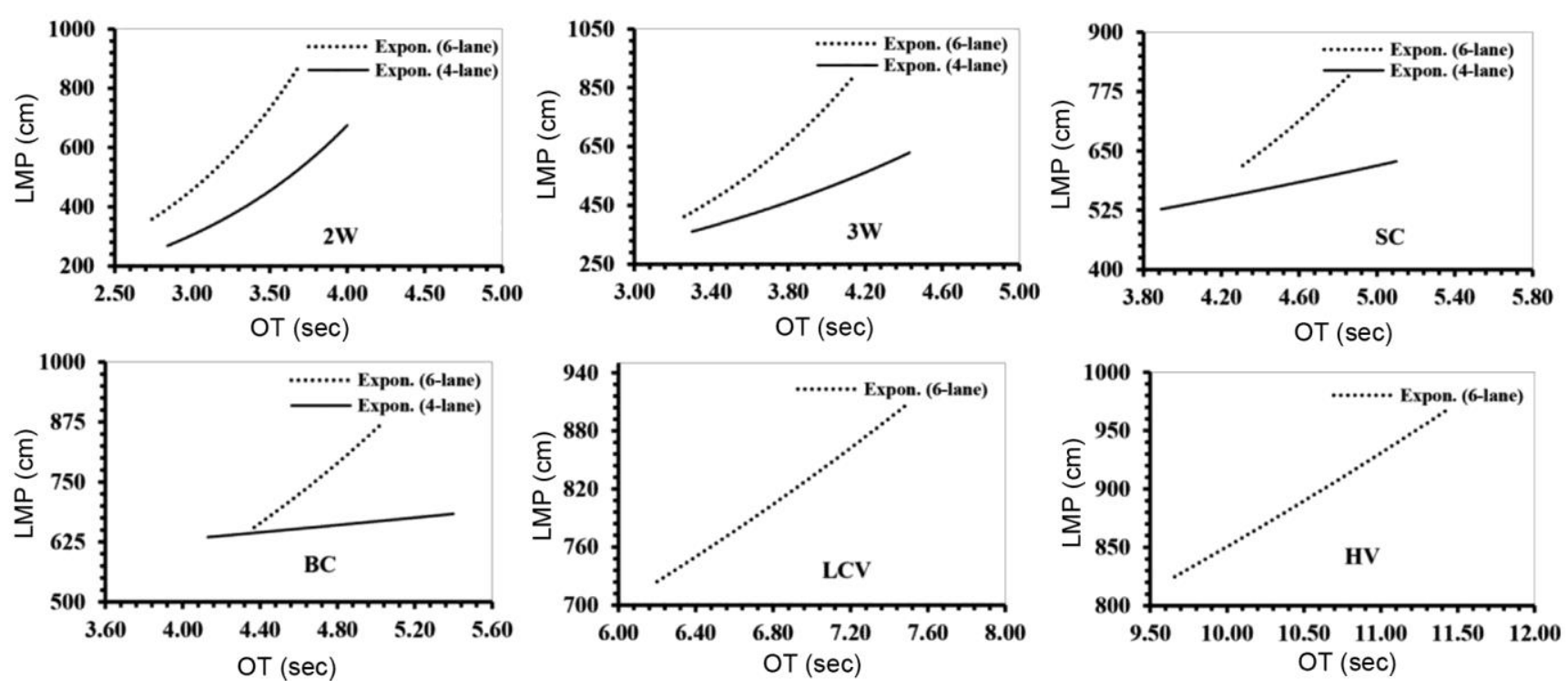

Figure 5. Disparity of $\mathrm{LWM}_{85}$ at varying occupancy time in six-lane and four-lane roads.

independent variables at a $95 \%$ confidence level (i.e. $P$ value $\leq 0.05)$. The models obtained for different categories of vehicles for six-lane and four-lane divided roads are given in eqs (3)-(8) and eqs (9)-(12) respectively.

$$
\begin{aligned}
& { }^{2 \mathrm{~W}_{\mathrm{LWM}}} \mathrm{LW}_{85}=971.676-0.113 \mathrm{ATTV} \text {, } \\
& \text { adjusted } R^{2}=0.90 \text {, } \\
& \text { adjusted } R^{2}=0.85 \text {, } \\
& \text { adjusted } R^{2}=0.83 \text {, } \\
& { }^{\text {вC }} \mathrm{LWM}_{85}-994.493-65.149 \mathrm{SD} \\
& \text { adjusted } R^{2}=0.78 \text {, } \\
& { }^{\mathrm{LCV}} \mathrm{LWM}_{85}=948.343-0.036 \mathrm{ATTV} \text {, } \\
& \text { adjusted } R^{2}=0.84 \text {, }
\end{aligned}
$$

$$
\begin{aligned}
{ }^{\mathrm{HV}} \mathrm{LWM}_{85}= & 990.653-0.028 \mathrm{ATTV}, \\
& \text { adjusted } R^{2}=0.73,
\end{aligned}
$$

$$
\begin{array}{r}
{ }^{2 \mathrm{~W}} \mathrm{LWM}^{85}=-679.44+332.17 \mathrm{OT}, \\
\text { adjusted } R^{2}=0.88, \\
{ }^{3 \mathrm{~W}} \mathrm{LWM}_{85}=-409.57+232.71 \mathrm{OT}, \\
\text { adjusted } R^{2}=0.76,
\end{array}
$$

$$
{ }^{\mathrm{SC}} \mathrm{LWM}_{85}=205.06+82.83 \mathrm{OT}
$$$$
\text { adjusted } R^{2}=0.61 \text {, }
$$

$$
\begin{array}{r}
{ }^{\mathrm{BC}} \mathrm{LWM}_{85}=477.29+38.17 \mathrm{OT}, \\
\text { adjusted } R^{2}=0.62 .
\end{array}
$$

The obtained models from stepwise multiple linear regression indicate that $\mathrm{LWM}_{85}$ of U-turning vehicles can be obtained from traffic operational characteristics such as ATTV, SD and OT. Equations (3)-(8) show the developed $\mathrm{LWM}_{85}$ prediction models for all the vehicles considered in six-lane roads. From eq. (3), it can be inferred that with increase in ATTV, $\mathrm{LWM}_{85}$ of $2 \mathrm{Ws}$ at the MBMO shifts towards the median side. A similar trend can be observed for the placement prediction model of $3 \mathrm{Ws}$ in a six-lane road. From eqs (5) and (6), it can be observed that SD has an influence on $\mathrm{LWM}_{85}$ of SCs and BCs for six-lane roads. As SD increases, $\mathrm{LWM}_{85}$ tends to shift towards the median opening. This can be due to the fact that drivers become impatient when waiting for a longer time at the median opening. Such impatient drivers try to merge aggressively by utilizing less width of the road. Similar observations were reported by Sil et al. ${ }^{23}$. $\mathrm{LWM}_{85}$ of LCVs and HVs can be predicted from ATTV as given in eqs (7) and (8). From these equations, it can be seen that their coefficients are small compared to that of $\mathrm{LWM}_{85}$ prediction model of $2 \mathrm{Ws}$ and $3 \mathrm{Ws}$. This is because that HVs are bigger in size and have a larger turning radius. Therefore, $\mathrm{LWM}_{85}$ is not affected much by variation of ATTV. Furthermore, from eqs (7) and (8), it can be inferred that if ATTV increases, the LWM decreases, i.e. LWM shifts towards the median side. Therefore, for six-lane roads, ATTV, SD and OT are essential parameters for the prediction of $\mathrm{LWM}_{85}$ of U-turning vehicles.

Likewise, $\mathrm{LWM}_{85}$ prediction models were developed for different categories of vehicles on four-lane roads. The developed models are presented in eqs (9)-(12). Due 
to the unavailability of sufficient data, models could be developed for LCVs and HVs. From eqs (9)-(12), it can be observed that $\mathrm{LWM}_{85}$ of all vehicle categories depends upon OT. Further, it can be inferred that $\mathrm{LWM}_{85}$ tends to shift towards the kerbside with increase in OT. Therefore, by calculating OT, it is possible to predict $\mathrm{LWM}_{85}$ of all vehicles in a four-lane road.

\section{Model validation}

For validating the proposed model, $\mathrm{LWM}_{85}$ of U-turning vehicles at the MBMOs obtained from the model was compared with that observed from field data. The field data were collected from two different MBMOs of sixlane and four-lane roads. These field data have not been used for developing the $\mathrm{LWM}_{85}$ models. For validating the $\mathrm{LWM}_{85}$ model, $\mathrm{LWM}_{85}, \mathrm{SD}$, OT and ATTV were estimated for all vehicles from the collected videos. Table 2 shows a comparison between $\mathrm{LWM}_{85}$ obtained from field data and the proposed models.

Table 2 shows that $\mathrm{LWM}_{85}$ values obtained from the model and field are in close agreement with each other. Moreover, the minimum error and maximum error in estimating the values are $7.57 \%$ for $\mathrm{HVs}$ and $13.54 \%$ for $\mathrm{SCs}$, for six-lane roads and $7.13 \%$ for $\mathrm{BCs}$ and $12.26 \%$ for $3 \mathrm{Ws}$ for four-lane roads. Therefore, the proposed models are efficient for predicting $\mathrm{LWM}_{85}$ for all the vehicles considered in the six-lane and four-lane roads.

\section{Implication of the study}

The U-turning vehicles give rise to conflict with ATT at some portions of the MBMO area, which many time lead to congestion. There is the presence of a virtual conflict zone at the MBMO area, and the dimensions of this zone depend on LWM and composition of the U-turning traffic stream. Therefore, by identifying the lateral and longitudinal dimensions of the conflict zone, a geometrical augmentation scheme can be proposed.

Table 2. Model validation

\begin{tabular}{lcccc}
\hline & & \multicolumn{2}{c}{ LWM $_{85}$} & \\
\cline { 3 - 4 } $\begin{array}{l}\text { Category of } \\
\text { vehicles }\end{array}$ & $\begin{array}{c}\text { Number of } \\
\text { lanes }\end{array}$ & $\begin{array}{c}\text { Proposed } \\
\text { model }\end{array}$ & $\begin{array}{c}\text { Field } \\
\text { observation }\end{array}$ & $\begin{array}{c}\text { Difference in } \\
\text { percentage }\end{array}$ \\
\hline 2W & Six-lane & 491 & 435 & 13.52 \\
3W & & 621 & 550 & 11.43 \\
SC & & 665 & 575 & 13.54 \\
BC & & 593 & 650 & 08.78 \\
LCV & & 795 & 725 & 08.84 \\
HV & & 855 & 925 & 07.57 \\
2W & & 560 & 500 & 10.71 \\
$3 W$ & & 508 & 579 & 12.26 \\
SC & & 548 & 600 & 08.67 \\
BC & & 673 & 625 & 07.13 \\
\hline
\end{tabular}

In an effort to identify the lateral dimension of the conflict zone, a simple but practical equation has been proposed below.

$$
W_{\mathrm{lw}}=\sum_{i=1}^{N} U_{i} * f_{i},
$$

where $W_{\mathrm{lw}}$ is the width of lateral widening, $U_{i}$ the proportion of different categories of U-turning traffic, $f_{i}$ the $\mathrm{LWM}_{85}(\mathrm{~cm})$ of $i$ th category of U-turning traffic and $N$ is the total number of different categories of traffic.

Estimation of $\mathrm{LWM}_{85}$ of different vehicle categories is among the challenging tasks in the implementation of geometrical augmentation scheme. $\mathrm{LWM}_{85}$ estimation requires extensive LWM data of U-turning vehicles. Furthermore, for LWM data collection, road marking and segmentation of carriageway are indispensable. Therefore, to ease the $\mathrm{LWM}_{85}$ estimation, $\mathrm{LWM}_{85}$ prediction models have been developed in this study. $\mathrm{LWM}_{85}$ for all vehicles considered in six-lane and four-lane roads is presented in eqs (3)-(12). For example, $\mathrm{LWM}_{85}$ of $2 \mathrm{Ws}$ in six-lane roads can be predicted using eq. (3), which requires ATTV. Utilizing ATTV of any section during the peak period, $\mathrm{LWM}_{85}$ of $2 \mathrm{Ws}$ for that particular section can be estimated. Likewise, utilizing the proposed equations, $\mathrm{LWM}_{85}$ of all the vehicle categories can be estimated utilizing ATTV and SD for six-lane roads and OT for four-lane roads. Therefore, the developed $\mathrm{LWM}_{85}$ models will be beneficial for traffic engineers to predict $\mathrm{LWM}_{85}$ of different vehicle categories. Based on the estimated $\mathrm{LWM}_{85}$ of different vehicle categories and composition of U-turning traffic, the required width of lateral widening at any MBMO can be estimated.

Further, we estimated the longitudinal length for which lateral widening is required at the MBMO area. At the MBMO region, the priority of approaching through vehicles is often compromised due to high traffic volume, as well as the impatient and discourteous behaviour of drivers of U-turning vehicles ${ }^{29}$. Due to the reversal of priority at the MBMOs, many a times, approaching through vehicles decelerate before entering the MBMO area. This slow-down section starts from the upstream end of the conflict zone and extends up to the merging line. To identify the slow-down section, brake light approaching through vehicles is observed in the field. The brake light glows when drivers remove their leg from the accelerator paddle, which signifies that the vehicle has started deaccelerating, or it is not at its desired speed. Moreover, significant differences in the slow-down section for different vehicle categories were observed in the field. From the field observations, the slow-down section can be calculated using the composition of ATTV (eq. (14)). The slow-down section will be different for different MBMOs depending upon the composition of the U-turning vehicles and ATTV. 


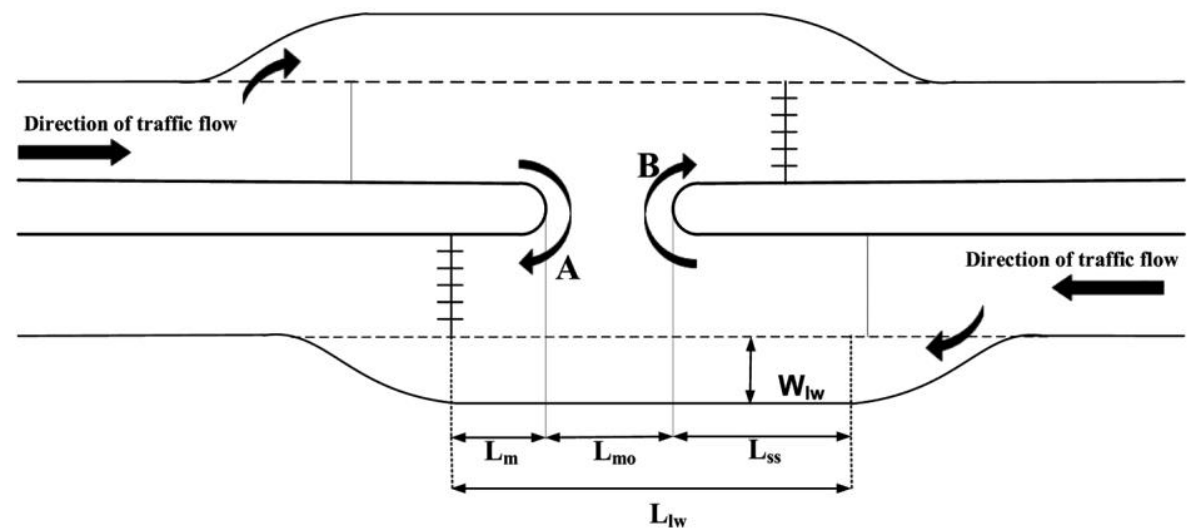

Figure 6. Schematic diagram of the proposed geometrical improvement.

$$
L_{\mathrm{lw}}=\sum_{i=1}^{N} T_{i} * L_{\mathrm{SS}},
$$

where $L_{\mathrm{lw}}$ is the length of lateral widening, $T_{i}$ the proportion of different categories of ATT, $L_{\mathrm{SS}}$ the length of slow-down section, and $N$ is the total number of different categories of traffic.

In the proposed geometrical augmentation scheme, the length for which extra widening is required is basically the distance from the start of the slow-down section to the merging line. Figure 6 provides details of the proposed geometrical augmentation scheme. This scheme targets to mitigate congestion in the MBMO area by providing extra space for vehicular traffic.

\section{Conclusion}

The present study was carried out to develop a model for LWM for different types of U-turning vehicles at the MBMOs on urban roads. The following conclusions can be made from this study.

(1) $\mathrm{LWM}_{85}$ decreases with increase in SD and ATTV (LWM ${ }_{85}$ shifts towards the median side), whereas it increases with an increase in OT $\left(\mathrm{LWM}_{85}\right.$ shifts towards the kerb).

(2) $2 \mathrm{Ws}$ experienced the lowest SD among all vehicle categories considered in this study, while HVs were found to experience the highest SD. Moreover, SD was also observed to vary depending on ATTV. Therefore, SD experienced by a U-turning vehicle in this study ranges from $2.33 \mathrm{sec}$ (for $2 \mathrm{Ws}$ at the lowest ATTV) to $6.44 \mathrm{sec}$ (for HVs at the highest ATTV) in six-lane roads. Further, SD experienced by U-turning vehicles ranges from $2.32 \mathrm{sec}$ (for $2 \mathrm{Ws}$ at the lowest ATTV) to $9.35 \mathrm{sec}$ (for BCs at the highest ATTV) in four-lane roads. It can be observed that SD experienced by U-turning vehicles is less at lower ATTV compared to higher ATTV, because in the former situation U-turning vehicles have to wait at the median opening for a longer time compared to higher traffic volume. Due to the long waiting period, U-turning vehicles try to merge swiftly using less width of the carriageway. Therefore, it can be inferred that the category of vehicles and ATTV are important factors which influence SD of U-turning vehicles.

(3) $2 \mathrm{Ws}$ were found to experience less OT among all vehicle categories considered in this study, and highest OT was observed for HVs. Furthermore, OT was found to fluctuate depending on ATTV. Therefore, OT of a U-turning vehicle in this study ranges from $2.74 \mathrm{sec}$ (for 2Ws at the highest ATTV) to $11.45 \mathrm{sec}$ (for HVs at the lowest ATTV) in six-lane roads. Further, OT ranges from $2.84 \mathrm{sec}$ (for $2 \mathrm{Ws}$ at the highest ATTV) to $5.40 \mathrm{sec}$ (for BCs at the lowest ATTV) in four-lane roads. It can be observed that OT is more at lower compared to higher ATTV, because in the former condition, U-turning vehicles have a tendency to merge freely using more width of carriageway but as ATTV increases U-turning vehicles try to squeeze towards the median opening to avoid collision with ATTV. Therefore, it can also be inferred that the category of vehicles and ATTV are important factors which influence OT of U-turning vehicles.

(4) SD experienced by U-turning vehicles was found to be higher in four-lane compared to six-lane roads at identical ATTV. This is because in four-lane roads, width of the carriageway is less compared to six-lane roads. Therefore, U-turning vehicles have to wait for a longer time to find a suitable gap for merging at the four-lane roads compared to six-lane roads. Likewise, OT of Uturning vehicles was found to be higher in four-lane roads compared to six-lane roads at identical ATTV. This is because U-turning vehicles merge gradually at four-lane roads, and thus accelerate and decelerate several times before merging. Additionally, the turning speed of fourlane vehicles is also less than that of six-lane vehicles.

(5) LWM can be predicted using SD and ATTV in sixlane roads, whereas only OT is required for predicting LWM for different types of vehicles in four-lane roads. 
The accuracy of the developed LWM model was found to be satisfactory as the maximum error was found to be around $13.5 \%$.

Based on the above-mentioned findings, a simple geometrical augmentation scheme has been suggested to reduce the congestion and improve the level of service and improve safety. The present study holds significance for efficient traffic planning, operation and design at the MBMO area in divided roads. The findings of this study can be utilized by traffic engineers and policymakers to improve traffic operating condition at the MBMO area not only in developing countries but also in developed countries with homogenous and lane disciplined traffic conditions.

1. GoI, Basic Road Statistics of India (Report), Ministry of Road Transport and Highways, Government of India, 2016.

2. Das, U., Trends in growth of roads development programmes in India. Int. J. Soc. Sci. Econ. Res., 2018, 3(6), 2617-2630.

3. Cooner, S. A., Rathod, Y. K., Alberson, D. C., Bligh, R. P., Ranft, S. E. and Sun, D., Development of guidelines for cable median barrier systems in Texas. Federal Highways Administrations, US Department of Transportation, USA, 2009

4. Mondal, S. and Gupta, A., Assessment of vehicles headway during queue dissipation at signal-controlled intersection under mixed traffic. Curr. Sci., 2019, 116(3), 437-444.

5. Mohapatra, S. S. and Dey, P. P., Lateral placement of U-turns at median openings on six-lane divided urban roads. Transp. Lett, 2015, 7(5), 252-263.

6. Saha, A., Chandra, S. and Ghosh, I., Assessment of level of service for urban signalized intersections in India. Curr. Sci., 2019 117(9), 1516.

7. Dey, P. P., Nandal, S. and Kalyan, R., Queue discharge characteristics at signalised intersections under mixed traffic conditions. Eur. Transp., 2013, 55(7), 1-12.

8. Aldian, A. and Taylor, M. A. P., Selecting priority junction traffic models to determine U-turn capacity at median opening. Eastern Asia Soc. Transp. Stud., 2001, 3(2), 101-113.

9. Khan, T. and Mohapatra, S. S., Effect of operational attributes on lateral merging position characteristics at mid-block median opening. Transp. Lett., 2020, 13(2), 1-14.

10. Liu, P., Lu, J. J., Zhou, H. and Sokolow, G., Operational effects of U-turns as alternatives to direct left-turns. J. Transp. Eng., 2007, 133(5), 327-334.

11. Cassidy, M. J., Madanat, S. M., Wang, M. H. and Yang, F., Unsignalized intersection capacity and level of service: revisiting critical gap. Transp. Res. Rec., 1995, 1484, 16-23.

12. Kaysi, I. and Alam, G., Driver behavior and traffic stream interactions at unsignalized intersections. J. Transp. Eng., 2000, 126(6), 498-505.

13. Brilon, W. and $\mathrm{Wu}, \mathrm{N}$., Capacity at unsignalized intersections derived by conflict technique. Transp. Res. Rec.: J. Transp. Res. Board, 2001, 1776, 82-90.

14. Al-Masaeid, H. R., Capacity of U-turn at median openings. Inst. Transp. Eng., ITE J., 1999, 69(6), 28-30.
15. Jenjiwattanakul, T. and Sano, K., Effect of waiting time on the gap acceptance behavior of U-turning vehicles at midblock median openings. J. East. Asia Soc. Transp. Stud., 2011, 9, 1601-1613.

16. Liu, P., Lu, J. J., Hu, F. and Sokolow, G., Capacity of U-turn movement at median openings on multi-lane highways. J. Transp. Eng., 2008, 134(4), 147-154.

17. Mohapatra, S. S. and Dey, P. P., Estimation of U-turn capacity at median openings. J. Transp. Eng., Part A, 2018, 144(9), 04018049

18. Liu, P., Qu, X., Yu, H., Wang, W. and Cao, B., Development of a VISSIM simulation model for U-turns at unsignalized intersections. J. Transp. Eng., 2012, 138(11), 1333-1339.

19. Zhang, S., Chang, Y. L. and Wu, Z. H., Probabilistic model of capacity of short right-turn lanes at signalized intersections. J. Transp. Syst. Eng. Inf. Technol., 2015, 15(4), 192-197.

20. Liu, P., Lu, J. and Cao, B., Capacity of U-turns at unsignalized median openings on six-lane streets. Transp. Res. Rec.: J. Transp. Res. Board, 2009, 2130, 59-65.

21. Mehar, A., Chandra, S. and Velmurugan, S., Speed and acceleration characteristics of different types of vehicles on multi-lane highways. Eur. Transp., 2013, 55(1), 1-12.

22. Khan, T. and Mohapatra, S. S., Lateral placement characteristics of U-turning vehicles: a statistical investigation. Transp. Develop. Econ., 2020, 6(1), 1-12.

23. Sil, G., Mohapatra, S. S., Dey, P. P. and Chandra, S., Merging process of U-turns at uncontrolled median openings under mixed traffic conditions. Transport, 2016, 33(2), 370-379.

24. Benjamini, Y., Opening the box of a boxplot. Am. Stat., 1988, 42(4), 257-262.

25. Day, C. M., Wasson, J. S., Brennan Jr, T. M. and Bullock, D. M., Application of travel time information for traffic management. Indiana Department of Transportation and Purdue University, West Lafayette, Indiana, USA, 2012.

26. Kanagaraj, V., Srinivasan, K. and Sivanandan, R., Modeling vehicular merging behaviour under heterogeneous traffic conditions. Transp. Res. Rec.: J. Transp. Res. Board, 2010, 2188, 140-147.

27. Fitzpatrick, K., Krammes, R. A. and Fambro, D. B., Design speed, operating speed and posted speed relationships. Inst. Transp. Eng., ITE J., 1997, 67(2), 52.

28. Ewadh, H. A. and Neham, S. S., Conflict to study safety at four leg-signalised intersections. Proc. Inst. Civ. Eng.-Transp., 2011, 164(4), 221-230.

29. Mohapatra, S. S., Dey, P. P. and Chandra, S., Conflicting volume for U-turns at uncontrolled median openings. Proc. Inst. Civ. Eng.-Transp., 2016, 169(4), 195-204

ACKNOWLEDGEMENTS. This study is part of an on-going Minor Research Project titled 'Effect of geometrical attributes of median opening on capacity of U-turn', funded by TEQIP-III. Financial assistance provided by the project is acknowledged.

Received 16 May 2020; revised accepted 28 March 2021

doi: $10.18520 / \mathrm{cs} / \mathrm{v} 120 / \mathrm{i} 11 / 1768-1777$ 